2021, Volume 8, ID 583

DOI: $10.15342 /$ ijms.2021.583

ORIGINAL RESEARCH

\title{
The Contribution of Lumbar Puncture in Neonatal Infections - About 206 cases
}

\author{
Kaoutar Ettoini, Yousra El Boussaadni iD, Abdallah Oulmaati \\ Pediatrics department, Mohamed V hospital, Faculty of Medicine and Pharmacy, University of AbdelmalekEssaadi-Tangier, \\ Tangier, Morocco
}

\begin{abstract}
Background: Neonatal meningitis is a serious infection. No clinical and biological score has been established to accurately identify neonates at high risk of developing neonatal meningitis.

Objective: The aim of this work is to clarify the place of lumbar punctures in neonatal infections and to identify the predictive factors of meningeal localization in case of neonatal infection.

Materials and methods: This is a prospective study of 861 observations of newborns hospitalized in the pediatric department of Mohammed V Hospital, CHU of Tangier, during a 14-month period from 1 January 2019 to 29 February 2020. Among these patients, the diagnosis of neonatal infection (NNI) was retained in 473 cases. Initial lumbar puncture was performed in 206 cases $(43 \%)$. We included neonates aged 0 to 28 days, suspected of NNI, who had a lumbar puncture. Neonates treated as carriers of neonatal infection without sufficient anamnestic and clinical evidence and with an inconclusive or unperformed biological workup were excluded from the study.

Results: During the study period, 861 newborns were hospitalized, and the diagnosis of neonatal infection was retained in 473 cases, a rate of 55\%, and the initial lumbar puncture was performed in 206 patients (43\%). 61 newborns were diagnosed with neonatal meningitis, with fever in $76 \%$ of cases, $85 \%$ with convulsions, hypotonia and/or refusal to suckle in $63 \%$ of cases, and $\mathrm{CRP}>25 \mathrm{mg} / \mathrm{l}$ in $67 \%$ of newborns.

Conclusion: Lumbar puncture is the only diagnostic means of meningitis. Indeed, the indication of this procedure should not be systematic, but it should be dictated by the careful and simultaneous analysis of the anamnestic, clinical and biological criteria evocative of the infection and its meningeal localization in order to diagnose meningitis early and treat it correctly. The need to establish scores combining these different parameters in order to accurately identify newborns at high risk of developing neonatal meningitis
\end{abstract}

KEYWORDS: Lumbar Puncture, Newborn, Neonatal Infections, Meningitis

Correspondence: Kaoutar Ettoini, Pediatrics department, Mohamed V hospital, Faculty of Medicine and Pharmacy, University of Abdelmalek Essaadi-Tangier, Tangier, Morocco. Email: ettoinik@gmail.com

Copyright (C) 2021 Ettoini $K$ et al. This is an open access article distributed under the Creative Commons Attribution 4.0

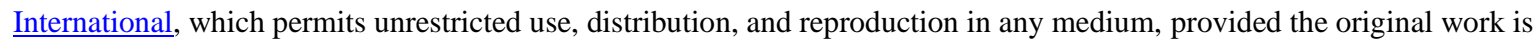
properly cited.

\section{BACKGROUND}

Neonatal bacterial infection (NBI) represents the first diagnosis evoked in any neonate who is unwell $[1,2]$. The prognosis of NBI is conditioned by the meningeal location, with a mortality rate between $37.7 \%$ and $66 \%$ [3], and $20 \%$ of survivors have neurological sequelae.

Lumbar puncture is the only means of diagnosing meningitis; failure to recognize a meningeal location is responsible for insufficient dosage and too short a treatment period, exposing the patient to the risk of ineffectiveness and psychomotor and neurosensory sequelae.

This work aims to review the place of lumbar punctures in neonatal infections and to identify the predictive factors of meningeal localization in case of neonatal infection.

\section{MATERIAL AND METHODS}

This is a prospective study of 861 observations of newborns hospitalized in the pediatric department of
Mohammed V Hospital, CHU of Tangier, for 14 months from January 2019 to 29 February 2020. Among these patients, the diagnosis of neonatal infection (NNI) was retained in 473 cases. To make the diagnosis of NNI minor and major anamnestic criteria of NNI, reported by the National Agency for Accreditation and Evaluation in Health (ANAES), clinical examination data, significant disturbances of CRP, and blood count were considered. We included neonates aged 0 to 28 days with suspected NBI who underwent lumbar puncture:

Patients with an NNI retained the presence of anamnestic and clinical criteria with a significant increase of inflammation markers.

$\square$ Patients with NNI based on the presence of anamnestic criteria but asymptomatic on examination, in whom the inflammatory workup showed significant disturbances unrelated to another pathology of the newborn.

Patients without minor or significant anamnestic elements favor a neonatal infection. Still, the second 
appearance of symptoms suggestive of the disease led to an inflammatory workup showing substantial disturbances.

$\square$ Patients with minor and/or significant criteria for neonatal infection with symptoms on clinical examination who were treated despite the absence of inflammatory markers at baseline.

Neonates treated as carriers of neonatal infection without sufficient anamnestic and clinical evidence with an inconclusive or unperformed biological workup were excluded from the study.

The biological arguments in favor of an infection are: Hyperleukocytosis (> 25,000/mm3) or leukopenia (< $5000 / \mathrm{mm} 3)$, thrombocytopenia $(<150,000 / \mathrm{mm} 3)$. At the level of the inflammatory workup: a CRP was considered positive if $\geq 25 \mathrm{mg} / \mathrm{L}$ was performed at 24 hours or 48 hours if the first CRP is normal. At the level of bacteriological assessment, positive blood culture, and CSF culture. The criteria of positivity of a lumbar puncture, which we retained, in this study, are:

o A cell response greater than or equal to 30 elements/mm3.

o Glucorachy to blood glucose ratio $<0.5$.

o Hyperproteinorachia $>=1.3 \mathrm{~g} / 1$.

o A positive direct examination after Gram stain.

o Positive culture or presence of soluble antigens in CSF.

All data were collected on an individual pre-established datasheet and are processed and analyzed by the SPSS software.

\section{RESULTS}

During the study period, 861 neonates were hospitalized with a diagnosis of neonatal infection in 473 cases, a rate of $55 \%$. The maternal and neonatal clinical characteristics of the study population are summarized in Table 1

In our series, the CRP is higher than $25 \mathrm{mg} / 1$ in $84 \%$ of cases (table 2)

Hyperleukocytosis $>=25000$ elements $/ \mathrm{mm}^{3}$ in $15 \%$ of cases with leukopenia $=<5000$ elements $/ \mathrm{mm}^{3}$ in $11 \%$.Positive blood culture in $6 \%$. The initial lumbar puncture was performed in 206 cases $(43 \%)$. It was completed before the start of antibiotic therapy in 20 neonates or $10 \%$, while 186 LPs were performed only after antibiotic treatment or $90 \%$. Sixty-one of the cases $(29.5 \%)$ had a positive LP, 136 cases $(66 \%)$ had a negative LP, and 10 LPs were traumatic (4.5\%). The germ was isolated in only two possibilities: salmonella and listeria. The diagnosis of neonatal meningitis was made in 61 neonates, of whom $76 \%$ had a fever, $85 \%$ had convulsions, $63 \%$ had hypotonia and refusal to suckle, and $67 \%$ had a CRP $>25 \mathrm{mg} / 1$ (Figure 1). 98\% of neonates with neonatal meningitis had a normal transcutaneous ultrasound in $93 \%$ of cases, and a brain CT scan was normal in $91 \%$ of cases. Management was symptomatic with antibiotic therapy prescribed in all newborns.

\section{DISCUSSION}

Neonatal meningitis corresponds to life-threatening disease and affects 0.1 to 0.4 neonates per 1,000 live births $[4,5,6]$. Its prevalence in industrial countries is lower, and it is $0.17 \%$ in the United States, $0.16 \%$ in France. A prevalence ranging from 2.4 to 6.1 per 1000 live births is observed in emerging countries [1].
Table 1: Maternal and neonatal clinical characteristics of the study population

\begin{tabular}{|c|c|c|}
\hline Clinical characteristics & $\mathbf{N}$ & $\%$ \\
\hline \multicolumn{3}{|l|}{ Gender } \\
\hline - Male & 176 & $37 \%$ \\
\hline - Female & 297 & $63 \%$ \\
\hline \multicolumn{3}{|l|}{ Gestational age } \\
\hline - $\quad<37$ WA & 80 & $17 \%$ \\
\hline - $\quad>37 \mathrm{WA}$ & 393 & $83 \%$ \\
\hline \multicolumn{3}{|l|}{ Progress of the pregnancy } \\
\hline - Pregnancy followed & 288 & $61 \%$ \\
\hline - Pregnancy not followed & 185 & $39 \%$ \\
\hline Medical delivery & 430 & $91 \%$ \\
\hline - $\quad$ vaginal delivery & 439 & $93 \%$ \\
\hline - cesarean section & 34 & $7 \%$ \\
\hline - Home delivery & 43 & $9 \%$ \\
\hline \multicolumn{3}{|l|}{ Rupture of the water pockets } \\
\hline - $\quad$ RWP> $12-18 \mathrm{~h}$ & 216 & $53 \%$ \\
\hline - $\quad$ RWP $<12 \mathrm{~h}$ & 190 & $47 \%$ \\
\hline \multicolumn{3}{|l|}{ The appearance of the amniotic fluid } \\
\hline - Clear liquid & 308 & $76 \%$ \\
\hline - $\quad$ colored liquid & 57 & $14 \%$ \\
\hline - $\quad$ pea-colored liquid & 41 & $10 \%$ \\
\hline \multicolumn{3}{|l|}{ Adaptation to ectopic life } \\
\hline - Apgar $<7$ at 5 th minutes & 80 & $15 \%$ \\
\hline - Apgar $>7$ at 5 th minutes & 393 & $85 \%$ \\
\hline Fever $\geq 38.5^{\circ} \mathrm{c}$ or hypothermia $\leq 36^{\circ} \mathrm{c}$ & 109 & $23 \%$ \\
\hline Heart rate $>160 \mathrm{c} / \mathrm{mn}$ & 19 & $4 \%$ \\
\hline CRT $>3$ seconds & 14 & $3 \%$ \\
\hline Respiratory rate $>60 \mathrm{c} / \mathrm{mn}$ & 160 & $34 \%$ \\
\hline Silvermann score $>4$ & 104 & $22 \%$ \\
\hline Refusal to feed & 212 & $45 \%$ \\
\hline Convulsions & 80 & $15 \%$ \\
\hline Bulging anterior fontanel (AF) & 24 & $5 \%$ \\
\hline Hypotonia & 322 & $68 \%$ \\
\hline
\end{tabular}

Table 2: CRP achieved in the study population CRP mg/l 24 hours of life After $48 \mathrm{~h}$ of Total

\begin{tabular}{lccc} 
& $\begin{array}{c}\text { (n/\%) } \\
\text { life }(\mathbf{n} / \%)\end{array}$ & \\
\hline Positive & $285 / 72$ & $112 / 28$ & $397 / \mathbf{8 4}$ \\
Negative & $53 / 70$ & $23 / 30$ & $76 / 16$ \\
Total & $338 / 71.5$ & $135 / 28.5$ & $473 / 100$ \\
\hline
\end{tabular}

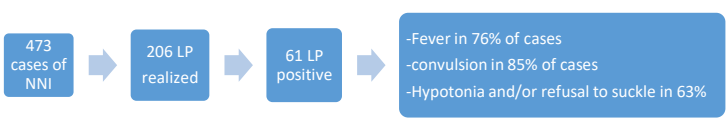

Figure 1: The overall synthesis of the results of our study

$10 \%$ of affected newborns die, and $20-50 \%$ of survivors develop hearing and visual impairments, seizures, motor abnormalities, and cognitive impairments [7].

Several studies have shown the involvement of two main germs in neonatal meningitis: streptococcus agalactiae (GBS) and E. coli. GBS accounts for 93\% of cases in term newborns and $35 \%$ of patients in premature infants in France, and E. coli is involved in $6 \%$ and $47 \%$, respectively. Listeria is found in $1.5 \%$ of cases, and meningococci and pneumococci in $1 \%$ of cases [1].

The clinical symptomatology of meningitis in newborns is not specific. It is in the form of a multitude of symptoms, including refusals to suckle, respiratory distress, thermal disturbances, convulsions, hypotonia, hemodynamic disturbances... the non-specificity of the clinical picture makes it difficult to distinguish between NBI with meningeal localization and other NBI [8].

In our study, we noted the presence of fever in $76 \%$ $(\mathrm{p}<0.005)$, convulsion in $85 \%(\mathrm{p}<0.005)$, hypotonia and refusal to suckle in $63 \%(\mathrm{p}<0.005)$, and a CCRP $>25 \mathrm{mg} / 1$ in $67 \%(p<0.005)$ of the neonatal meningitis cases. 
The diagnosis of bacterial meningitis is based on cerebrospinal fluid (CSF) culture [6]. However, the decision to perform lumbar puncture and the optimal time to do it in a newborn with signs of NBI is complex, and uncertain the non-specificity of clinical picture complicates this decision $[9,10]$.

Hemodynamic and respiratory instability or severe thrombocytopenia temporarily contraindicates lumbar puncture [1].

Delay in performing LP and placing neonates on broadspectrum antibiotics influences the interpretation of CSF results. In these situations, clinicians rely on interpreting CSF parameters such as glucose, cell counts, and protein levels to diagnose meningitis presumptively. Other factors that influence the interpretation of LP include gestational age, trauma causing CSF contamination with blood experienced during LP, and postnatal age [11-16].

Adequate treatment depends on a definite diagnosis; the definitive diagnosis of meningitis is made only by cerebrospinal fluid (CSF) culture. Many physicians choose to take a blood culture and wait for a positive result before performing a lumbar puncture, which underestimates the incidence of meningitis. In addition, 50\% of low birth weight $(<1500 \mathrm{~g})$ neonates with meningitis have a negative blood culture $[17,18]$.

\section{AUTHORS' CONTRIBUTIONS}

All the authors have actively participated in the redaction, the revision of the manuscript, and provided approval for this final revised version.

\section{REFERENCES}

[1] Chemsi M, Elmasbahi F, Lami AS, Lehlimi M, Habzi A, Benomar S. La ponction lombaire dans l'infection néonatale bactérienne précoce : Performance et décision. J Pédiatrie puériculture. 2018 Mar; 31(1): 27-33. Doi: 10.1016/i.jpp.2017.11.002

[2] Edmond K, Zaidi A. New approaches to preventing, diagnosing, and treating neonatal sepsis. PLoS Med. 2010 Mar 9; 7(3):e1000213. Doi:10.1371/journal.pmed.1000213

[3] Ku Lawrence C, Kim Boggess A, Cohen-Wolkowiez M. Bacterial Meningitis in Infants. Clin Perinatol. 2015 Mar; 42(1):29-45. Doi: 10.1016/j.clp.2014.10.004

[4] Okike IO, Johnson AP, Henderson KL, Blackburn RM, MullerPebody B, Ladhani SN, et al. Incidence, etiology, and outcome of bacterial meningitis in infants aged $<90$ days in the United kingdom and Republic of Ireland: prospective, enhanced, national population-based surveillance. Clin Infect Dis (2014) 59:e150-7. Doi: $10.1093 / \mathrm{cid} / \mathrm{ciu} 514$

[5] Lawn JE, Cousens S, Zupan J, Lancet Neonatal Survival Steering Team. 4 million neonatal deaths: when? where? why? Lancet. 2005 Mar 5-11; 365(9462):891-900. Doi: 10.1016/s01406736(05)71048-5

[6] Gordon SM, Srinivasan L, Harris MC. Neonatal Meningitis: Overcoming Challenges in Diagnosis, Prognosis, and Treatment with Omics. Front Pediatr. 2017 Jun 16; 5:139. Doi: 10.3389/fped.2017.00139

[7] Mann K, Jackson MA. Meningitis. Pediatr Rev. 2008 Dec; 29(12):417-29. Doi: 10.1542/pir.29-12-417

[8] Beam KS, Laughon MM, Hornik CP, Cohen-Wolkowiez M, Clark $\mathrm{RH}$, Benjamin DK Jr, et al. Predictors of Positive Cerebrospinal Fluid Cultures in Infants With Bacteremia. Pediatr Infect Dis J. 2014 Apr;33(4):360-5. Doi: 10.1097/inf.0000000000000115

[9] Jean-Baptiste N, Benjamin DK Jr, Cohen-Wolkowiez M, Fowler VG Jr, Laughon M, Clark RH, et al. Coagulase-negative staphylococcal infections in the neonatal intensive care unit. Infect Control Hosp Epidemiol. 2011 Jul; 32(7):679-686. Doi: $\underline{10.1086 / 660361}$
We were not able to perform blood cultures in all our cases, but we noted a CRP $>25 \mathrm{mg} / 1$ in $84 \%$ of the newborns with meningitis $(\mathrm{P}<0.005)$

Five parameters emerged from our study to predict the existence of a meningeal localization of neonatal infection. Through univariate analysis, a statistically significant relationship was established between the presence of fever, convulsion, hypotonia, and refusal to suckle and a CRP> $25 \mathrm{mg} / \mathrm{l}$ and, on the other hand, the positivity of the lumbar puncture and the diagnosis of meningitis.

\section{CONCLUSION}

Lumbar puncture is the only diagnostic means of meningitis; deciding not to perform a lumbar puncture in the newborn suspected of neonatal bacterial infection is not easy. Indeed, it must consider the risks incurred for the child both in terms of diagnosis and prognosis.

The indication of this procedure should not be systematic. Still, it should be dictated by the careful and simultaneous analysis of the anamnestic, clinical and biological criteria evocative of the infection and its meningeal localization to diagnose meningitis early and treat it correctly.

It's the need to establish scores combining these different parameters in order to accurately identify newborns at high risk of developing neonatal meningitis.

\section{COMPETING INTERESTS}

The authors declare no competing interests with this study.

\section{FUNDING SOURCES}

None.

[10] Stoll BJ, Hansen N, Fanaroff AA, Wright LL, Carlo WA, Ehrenkranz RA, et al. Late-onset sepsis in very low birth weight neonates: the experience of the NICHD Neonatal Research Network. Pediatrics. 2002 Aug; $110(2$ pt 1):285-91. Doi: 10.1542/peds.110.2.285

[11] Srinivasan L, Shah SS, Padula MA, Abbasi S, McGowan KL, Harris MC. Cerebrospinal fluid reference ranges in term and preterm infants in the neona tal intensive care unit. J Pediatr. 2021 Oct; 161 (4):729-34. Doi: 10.1016/j.jpeds.2012.03.051

[12] Greenberg RG, Benjamin DK, Cohen-Wolkowiez M, Clark RH, Cotten CM, Laughon M, et al. Repeat lumbar punctures in infants with meningitis in the neonatal intensive care unit. J Perinatol. 2011 Jun; 31(6):425-9. Doi: 10.1038/jp.2010.142

[13] Garges HP, Moody MA, Cotten CM, Smith PB, Tiffany KF, Lenfestey R, et al. Neonatal Meningitis: What Is the Correlation Among Cerebrospinal Fluid Cultures, Blood Cultures, and Cerebrospinal Fluid Parameters? Pediatrics. 2006 Apr; 117(4):1094-100. Doi:10.1542/peds.2005-1132

[14] Smith PB, Garges HP, Cotton CM, Walsh TJ, Clark RH, Benjamin DK. Meningitis in preterm neonates: importance of cerebrospinal fluid parameters. Am J Perinatol. 2008 Aug; 25(7):421-6. Doi: 10.1055/s-0028-1083839

[15] Srinivasan L, Harris MC, Shah SS. Lumbar puncture in the neonate: challenges in decision making and interpretation. Semin Perinatol. 2012 Dec; 36(6):445-53. Doi: $10.1053 /$ j.semperi.2012.06.007

[16] Srinivasan L, Shah SS, Abbasi S, Padula MA, Harris MC. Traumatic lumbar punctures in infants hospitalized in the neonatal intensive care unit. Pediatr Infect Dis J. 2013 Oct; 32(10):1150-2. Doi:10.1097/inf.0b013e31829862b7

[17] Harvey D, Holt DE, Bedford H. Bacterial meningitis in the newborn: a prospective study of mortality and morbidity. Semin Perinatol. 1999 Jun; 23(3):218-25. Doi: 10.1016/s01460005(99)80066-4

[18] Stoll BJ, Hansen N, Fanaroff AA, Wright LL, Carlo WA, Ehrenkranz RA, et al. To tap or not to tap: high likelihood of meningitis without sepsis among very low birth weight infants. Pediatrics. 2004 May; 113(5):11811186. Doi: $10.1542 /$ peds.113.5.1181 